\title{
SOME CONSIDERATIONS
}

WITH RESPECT

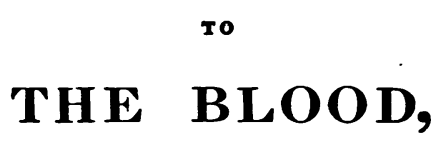

FOUNDED ON ONE OR TWO VERY SIMPLE EXPERIMENTS ON THAT FLUID.

BY BENJAMIN G. BABINGTON, M.D. F.R.S.

Read Jan. 25, 1830.

EXPERIMENT I.

LET blood be drawn in a full stream from the vein of a person labouring under acute rheumatism into a glass vessel which should be filled to the brim. By close inspection a colourless fluid will be immediately perceived around the edge of the surface, and after a rest of four or five minutes, a bluish appearance will be observed forming an upper layer on the blood, which is owing to the subsidence of the red particles to a certain distance. below the surface, and the consequent existence of a clear liquor between the plane of the red particles and the eye. Let now a spoon previously moistened with water, be carefully immersed into the upper layer of liquid by a gentle depression of one border. The liquid may thus be collected 
quite free from red particles, and will be found to be an opalescent and somewhat viscid solution perfectly homogeneous in appearance. By repeating the immersion we may collect this fluid in quantity and transfer it to another vessel. That which I employed, is a bottle holding about 180 grains, of globular form, with a narrow neck and perforated glass stopper.

Hewson went so far as to gather a small portion of this upper layer into a teaspoon, and as he found it after an interval to coagulate into a jelly, he concluded it to be coagulable lymph, and hence, in reasoning on the buffed coat of blood, thought the fibrine became specifically lighter than usual. Dr. Scudamore evidently has the same idea, when he states that liquid fibrine is lighter than serum. Hey thought that in buffed blood the different constituents were more intimately mixed together than in other cases, thus evincing his opinion of their separate existence; and Dr. Bostock in discussing the question whether the specific gravity of fibrine is increased by coagulation, evidently considers it as certain that pure fibrine exists in two states. We are led to the same conclusion with regard to his opinion when he speaks of fibrine as being " added to the blood particle by particle, so that while this fluid is in agitation in the vessels, it has no opportunity of coalescing or concreting." 
The sequel of the experiment just described clears up the errors on this point. The solution with which the globular bottle is filled though quite homogeneous at the time it is thus collected, is found after a time to separate into two parts, namely, a clot of fibrine which has the precise form of the bottle in which it was gathered, and a clear serum possessing all the usual characters of that fluid.

Hewson, who has led the way to the ascertainment of these facts, missed the right conclusion respecting them, by collecting so small a quantity of the fluid in question, and in so shallow a ressel that no separation was visible. It is, however, certain from the foregoing experiment, that buffed blood consists of only two constituents, namely, the red particles, and a liquid which I shall call Liquor Sanguinis.

It had long been remarked, that what is usually called inflamed blood, coagulated slower than healthy blood, and that the last portions of blood drawn from an animal which was bleeding to death coagulated quickest. The immediate cause of a buffy crust appears therefore to be as follows. The blood consisting of Liquor Sanguinis and insoluble red particles, preserves its fluidity long enough to permit the red particles, which are of greater specitic gravity, to subside through it. At 
length the Liquor Sanguinis separates, by a general coagulation and contraction, into two parts, and this phenomenon takes place uniformly throughout the liquor. That part of it through which the red particles had time to fall, furnishes a pure fibrine or buffed crust, while that portion into which the red particles had descended, furnishes the coloured clot. This, in extreme cases, may be very loose at the bottom, from the great number of red particles collected there, each of which has supplanted its bulk of fibrine, and consequently di. minished its firmness in that part. There is, however, with this limitation, no more fibrine in one part of the blood than another*.

- From the foregoing aecount of the cause of a buffed surface on blood, it will be easily perceived why the form of the ressel in which it is received influences this phenomenon-a matter of common observation. The space left by the gravitation of the red particles, bears a proportion to the whole perpendicular depth of the blood, so that in shallow vessels scarcely any buffed coat may appear where the same blood in a deep vessel would have furnished such a coat of considerable thickness. It is a fact, however, which I have not seen noticed, that the quantity of crassamentum is also dependent, within certain limits, on the form of the vessel employed. If this be shallow, thecrassamentum will be abundant, if approaching in form the cube or sphere, it will be scanty. This difference is owing to the greater or less distance of the coagulating particles of fibrine from a common centre, which causes a more or less powerful adhesion and contraction of those,particles. Perhaps few facts relating to the phenomena of renesection, are of more practical importance than this ; since blood is said to be thick or thin, rich or poor, in reference to the quantity of crassamen- 
It may be objected to a general conclusion drawn from the above experiment, that it is made upon blood in a diseased state. To obviate this objection, I have sought a means of placing healthy blood under such conditions as should enable its red particles to settle more quickly than would otherwise be the case.

tum it contains, and views of a disease are founded on these supposed conditions, which after all depend, not on the blood itself, but on the vessel into which it is received.

In the following examples the blood of one and the same venesection was received partly into a specific gravity bottle of a pear shape, and partly into a common pint basin. It was suffered to run into the one and the other vessel for several alternations, till both were charged to the extent required, thus obviating the possibility of difference in quality. After a day's rest, the vessels with their contents were weighed; the serum was then decanted from the clot, which with the vessels were again weighed; lastly the respective clots were removed, and the empty vessels were weighed. This simple method determined the weight of serum and clot in both cases.

Example I. An elderly man labouring under Purpura being bled, his blood at $87 \mathrm{Fah}$. had a specific gravity, 1050. Its serum at 65, 1027 of a deep yellow colour. The crassamențum was not cupped but was covered with a gelatinous translucent buff. Some of this blood being received into the pear shaped bottle, the proportion of serum to crassamentum was as 1000 to 1495 ; another portion being canght in a pint basin, its serum was to its crassamentum as 1000 to 2230.

Example II. A female aged twenty-four was bled for vertigo while pregnant. Her blood at 60 had a specific gravity 1049. Its serum at the same temperature 1028. The crassamentum was florid; neither firm nor buffed. Part of her blood being received into the pear-shaped bottle, its serum was to its 


\section{EXPERIMENT II.}

Take two similar tall glass jars or phials, each capable of holding about four or five ounces, and let one of them be half filled with olive oil; draw the blood of a healthy subject into each. That which flows through the oil will be found to have

crassamentum as 1000 to 945 , another part being received into the pint basin, its serum was to its crassamentum as 1000 to 1716.

Case III. A middle aged man with Phthisis; pulse 120, hard ; specific gravity of blood at 87,1044 ; serum at 60,1028 ; clot firm, with a bluish white, but not very thick buff. Serum to clot, in the bottle, 1000 to 960 , in the basin, 1000 to 1090.

Case IV. A female aged 35, labouring under Diabetes mellitus; specific gravity of blood, at 90,1048 ; specific gravity of crassamentum, 1079; of serum at 60,1024.6, as opaque, and nearly as white as milk. The clot was covered with a remarkably firm white crust, so tough, that when drawn over the mouth of a tumbler, after being freed from the red subjacent part, it dried into a membrane as thick as an ox's bladder. The remainder of the clot was very loose, and on being stirred about, became diffuent. Serum to clot in the pear-shaped bottle, as 1000 to 1292 , in the basin, as 1000 to $171 \%$.

I shall not, of course, be understood to affirm that in these cases the actual quantity of fibrine varied according to the vessel in which the blood was received. The difference is sufficiently explained, by supposing that the clot in the bottle is more compact and less spongy, and consequently contains a smaller proportion of serum in its insterstices than that formed in the basin. 
a layer of Liquor Sanguinis on its surface, which will form a buffed crust, while there will be none upon that which is received in equal quantity, and in other respects under the same circumstances into the empty jar. This experiment will not always succeed, for blood sometimes coagulates so quickly, even though it pass through oil, that no buffed crust is formed. It is enough, however, for my purpose, that there is frequently a very evident difference. If this experiment be performed on blood disposed to exhibit a buffy crust, that which is formed under the oil will be twice or thrice as thick as that formed in the empty jar. The reason that blood is buffed when drawn into oil we need not now consider. It is, indeed, sufficiently evident.

Healthy blood then is similarly constituted as blood disposed to form a buffed crust, the only difference being that the former coagulates more quickly than the latter.

From experiment the first, we may deduce in another manner that the Liquor Sanguinis is a uniform homogeneous fluid, and no mere mixture of fibrine and serum. The globular bottle with its perforated stopper, when perfectly filled with the liquor, exhibits on the separation of this fluid a clot which is precisely of the same shape (even including the indentation in the bottom,) as the 
vessel in which it has formed *. Hence, we may conclude, that coagulation took place uniformly from every part of the fluid, and the uniform density of the clot confirms this conclusion. Had the particles of the fibrine been in the least degree lighter than those of the serum, as supposed by Dr. Scudamore, the lower part of the clot would necessarily have been defective in form and density; so also, had the fibrine been heavier, the upper portion would have been similarly defective. Another circumstance which $I$ have ascer. tained by means of the globular bottle is, that no change takes place in the sum of the densities of the constituents of the Liquor Sanguinis by their separation, but that what the fibrine gains in specific weight is exactly balanced by what is lost by the serum. This is proved by the fact, that the bottle being exactly filled with Liquor Sanguinis at a given temperature remains full, notwithstanding the subsequent coagulation, as long as that temperature is maintained : whence it may be regarded as improbable that any free caloric is given out during the process of coagulation, since what the fibrine loses in capacity for heat by condensation, will in all likelihood be gained by the albuminous fluid. It is already known that albumen does not alter its volume on coagulation by heat; but I am not aware that this point has been hitherto investi-

* The clot of blood drawn into any mould, however varied in shape, takes its form with great exactness. 
gated with regard to the spontaneous coagulation which takes place in Liquor Sanguinis, or blood.

From the above experiments I am led to believe that fibrine and serum do not exist as such in circulating blood, but that the Liquor Sanguinis when removed from the circulation, and no longer under the influence of the laws of life,-has then, and not till then, the property of separating into fibrine and serum. This separation which may be considered a death of the blood, may, under disease, take place within the body, but never, as I think, consistently with healthy action.

It follows from the view here taken of the constitution of the blood, that there is no such animal fluid in existence as coagulable lymph. The Liquor Sanguinis cannot with propriety be so considered, for it is essentially a fluid, and if under certain circumstances it separates into two parts, only one of these (fibrine) is coagulable, nor can I admit that this one part, considered by itself, previously existed in a fluid state, for in order to its fluidity it was necessary that the two constituents should be so united as to form one compound. There is, therefore, no better reason for affirming, that fibrine exists in a fluid state in $\mathrm{Li}$ quor Sanguinis, than for affirming that muriatic acid exists in a solid state in muriate of ammonia. The salt, indeed, is solid of which muriatic acid 
forms one ingredient, but the ammonia is essential to the solidity of the compound. In like manner, the compound is fluid of which fibrine forms one ingredient, but the serum is essential to its fluidity.

Nor is it an unimportant error to suppose a fluid secreted from the blood which has the property of becoming converted into a solid, for we are thus led to overlook altogether the fluid portion of the compound with the albumen contained in it, which always forms by far the greater portion of the secretion.

The fact that healthy circulating blood consists of a homogeneous liquor and red particles, leads to another of much importance, which I believe will be found true in most cases, namely, that when an effusion of serum takes place, we shall generally, in some neighbouring part, find a corresponding deposition of fibrine. We are told, indeed, that serum is a secretion intended for the lubrication of closed membranes, as those of the ventricles of the brain, of the pericardium, of the peritoneum, \&cc. but this I venture to think is as erroneous a notion as that which supposes the existence of coagulable lymph. The fallacy is founded on appearances observed after death, which do not exist during life. When we recollect how quickly the separation of Liquor Sanguinis into serum and fibrine takes place out of the body, we 
ought not to be surprised to find, though it be but a few minutes after death, or even before it, if dissolution be gradual, a serous effusion into cavities which, during health, could not be destined to contain any fluid.

I doubt the fact, however, that such membranes have the power during health of secreting serum, by which term I mean a fluid essentially containing albumen; or that any thing more passes from them than an aqueous halitus, or vapour; and I therefore doubt the propriety of giving them the denomination of serous membranes. Under morbid defect of vitality they may and do suffer serum to exude from them containing more or less albumen, and in such cases we shall usually find effused into some neighbouring part the corresponding fibrine, which with the serum in question went to form the Liquor Sanguinis. Such membranes may pour forth the Liquor Sanguinis itself, in which case we shall find the separation to have taken place in the cavities which they line. Gelatinous masses will gravitate to their lowest parts, or flakes, or shreds of fibrine will be diffused through the fluid.

Wherever this gelatinous formation exists, it is owing to the presence of fibrine, since, as is well known, albumen never assumes a gelatinous form, under the ordinary temperature. We may indeed 
with albumen, when mixed with water and heated, exactly imitate this appearance of fibrine, and form substances of all degrees of gelatinous consistence; but this only serves to confirm the belief that fibrine, in a diluted state, may put on a gelatinous appearance on coagulation. I have stated that closed membranes may pour forth serum or Liquor Sanguinis. They may also, under high excitement, pour forth blood itself. There is, therefore, no better reason for calling such membranes serous, than for calling them fibrinous or sanguineous membranes. The secretion of each is morbid, and we ought not to designate parts from the morbid actions which may be set up in them.

The examination of fluids effused into closed cavities fiom diseased action, throws much light upon this subject. The fluid found in the ventricles of the brain in cases of hydrocephalus, usually contains so little albumen, that it does not coagulate on the application of heat. I have examined some specimens, the specific gravity of which scarcely exceeded that of distilled water. It generally varies from 1005 to 1010 ; I have found it as low as 1000.5; and only in one instance of acute inflammation of the brain, did it amount to 1019. Now I have invariably observed, that where the specific gravity is low, and there is consequently but little albumen contained in the fluid, little or. 
no gelatinous effusion will be found under the pia mater. Where, on the contrary, the specific gravity is high, the gelatinous effusion is very abundant, particularly at the basis of the brain*, and other marks of fibrinous deposition are visible.

In effusions into the chest, the specific gravity of the fluid is generally much higher than that of the ventricles of the brain, varying from 1019 to 1024.

From this circumstance I should a priori infer that a more abundant effusion of fibrine would be found somewhere to correspond with the albumen thus indicated. The result proves the accuracy of this inference. A thick false membrane, composed exclusively of fibrine, often lines the pleura. Fluid from the chest is sometimes clear, but oftener loaded with fibrine, sometimes suspended in flakes, sometimes in particles so fine, as to give it the appearance of cream ; after a few hours' rest these particles subside, and the clear serum in which they were suspended appears above. The fluid in this state may be justly called pus, which I believe to

* I am aware that a gelatinous appearance in the membranes of the brain, is sometimes owing to a mere infiltration of serum among the meshes of the cellular tissue, but $I$ have reference above to such a deposition of gelatinous substance as remains consistent after repeated perforation with a point or division with the blades of scissors. 
be no other than serum, in which minute particles of coagulated fibrine are suspended*. I have known such a secretion poured forth daily from the pleura, for more than a year together, preserving all along the same character and specific gravity.

What the circumstances are which determine the mode of separation of the fibrine from the serum, so that at one time it should form a false membrane adhering to the true membrane whence it exudes, at another, it should continue suspended in the serum itself, remains for future investigation. I shall, presently, offer some further remarks on this subject.

That such false membranes are formed of fibrine, resembling in every respect the buffed coat of blood, is very distinctly and beautifully shewn by drying portions of each, whilst stretched across the mouth of a small aperture, like the head of a drum. Not only will the identity of false membrane with the buffed crust of blood be thus demonstrated, but also the resemblance of both to true membrane be rendered apparent.

* This belief is negatively confirmed by the microscopical observations of Dr. Hodgkin, who says, in his paper on this subject, " as far as we bave yet examined this secretion, its particles appear to be as irregular in size and figure as those of the brain, and bear no resemblance to those of the blood." 
In ascites the effused fluid generally has a specific gravity of from 1014 to 1026 , and in proportion as it is charged with albumen, may we expect to find fibrine either diffused through it in gelatinous masses, flakes, or shreds; or otherwise forming the fibrinous base of some.preternatural growth, or false membrane lining the peritoneum. In peritonitis this last appearance is more especially evident.

In anasarca the gelatinous appearance of the cellular membrane from interstitial deposition is familiar to all; an appearance which as I have already observed, cannot depend on the presence of albumen. A section of the scrotum in those who have died under this state of disease, exhibits, very plainly, a gelatinous deposition of this kind. It will thus be perceived, that the part will not collapse, as it necessarily would, if the cellular membrane were merely infiltrated with serum, since the fluid form of this infiltration, and the ready communication of the interstices which contained it, would at once admit of its exit.

In the application of a blister we are furnished with another apt opportunity of witnessing the phænomena, which I conceive usually occur. The cutaneous vessels are excited; Liquor Sanguinis exudes from them, and separates the cuticle; serum is effused. Some fibrine is found in the most 
depending parts of the vesicles, while the principal part is deposited on the surface, and in a short time is condensed again to form a new cuticle.

The vital conditions on which the nature of the effusion of blood or its constituents depends, the present state of our knowledge does not enable us to determine. On the physical conditions, however, we may be permitted to offer some remarks. These respect the containing vessels, and the contained fluid. All that seems necessary on the part of the vessels in order to the production of an effusion, is an opening of their pores, so as to admit of a transudation; and it is clear, that solid particles of appreciable size would require larger apertures for their transudation than homogeneous fluid, and that a less patulous state would suffice for the passage of vapour. These three degrees would be marked by vaporous effusion, liquid effusion, and effusion of blood with its red particles, and all these effusions may, I think, actually occur. The production of pure water would be the result of the first degree. I have already said that this sometimes occurs in the ventricles of the brain, and it also probably takes place in other closed cavities, perhaps, even in a state of health. This water may, I conceive, be separated by exhalation in the form of vapour. There is no other method by which water can be separated from a solution, but by distillation. The only cause of the formation of 
vapour, at least the only one which can act in the body, is heat; an increased degree of heat, would, certainly, out of the body, produce an increased formation of vapour from any solution, and probably does so within its cavities. This vapour may find its way through pores which would not admit the transudation of any less subtle agent. We can, therefore, conceive a case of dropsy in which water might be accumulated without the transudation of any liquid whatever; being the consequence of only such a degree of expansion of the vessels as should admit the passage of vapour, and such a degree of local determination as should produce a preternatural local heat. Such a dropsy we should expect to find of chronic character ; since the formation of fluid by distillation at a temperature so low, and where the temperature of the cavity which acts the part of a receiver, must be so very little below that of the fluid from whence the vapour rises, must necessarily be a work of time. It appears to me possible, that in those cases of hydrocephalus where the specific gravity of the fluid scarcely exceeds that of distilled water, such may be the process by which the fluid is formed. I must not be understood to affirm it as a fact proved, that exhalation is one mode by which effusion is effected, but only to offer it as affording a possible explanation of those cases where nearly pure water is found. The fact that there is no serous effusion in these 
cases, taking the word serum to mean a fluid essentially containing albumen, I have not seen noticed; and yet nothing is to me more certain, than that death may be caused by a pure hydrocephalus, where there is no serous effusion, an assertion which would seem paradoxical, unless coupled with the explanation here offered.

Where there takes place an effusion of liquid, as contradistinguished from the supposed case of an exhalation of vapour, this may either consist of the Liquor Sanguinis, or of albuminous fluid. If of the latter, it either supposes a separation of the Liquor Sanguinis into fibrine and serum within the vessels previous to the effusion, which is probably of rare occurrence, or a secretion of serum, which we shall presently consider; but if the effusion be of Liquor Sanguinis, then it will vary in its character according to the degree of tenacity with which this liquor maintains its fluidity.

We may conceive a separation at the instant of effusion, and in consequence, an immediate formation of a minute, yet separate portion of fibrine and of serum, thus forming a liquid identical with pus. We may conceive on the other hand, that, previous to coagulation, time shall be allowed for the union of such transuded globules of Liquor Sanguinis in a fluid form, and according to the circumstances and locality under which this is ef- 
fused, that we may find gelatinous or fibrinous deposition or false membrane. Its subdivisions by the meshes of the cellular tissue and its union by effusion with previously organized structures, would also be additional causes of diversity in its characters, and if we go further, and suppose that after effusion and separation either its liquid or its solid portion is again taken up by the absorbents, we shall have yet another source of diversity in the product which will be found.

It may at first view seem at variance with our experience, that any deposition of fibrine should exist even in some of the most familiar instances of serous effusion. The surgeon immediately directs his mind to a hydrocele, and asks where is the evidence of deposition? I have certainly not had an opportunity of verifying my notion in this complaint, but it will diminish incredulity if $I$ here state in what proportion we are to expect to find fibrine, or how much we are called upon to account for. I may state as a general average * of the proportion of solid albumen in blood 6.00 out of 100 , and of fibrine 0.270 . Now the average specific gravity of healthy serum is 1029, and when, therefore, an effusion amounts to this

* This average is deduced from seventy-eight cases giren by $M$. Denis, in which the blood seems to have been very carefully analysed; see Recherches Experimentales sur le Sang Humain, considéré à l'Etat Sain, par P. S. Denis, 1830. 
strength, we may conclude that the quantity of fibrine deposited should be 0.27 part out of 100 . Applying this law to the cases of hydrocele, let us suppose eight ounces of serum to be drawn off of specific gravity 1029. We ought then to find fibrine deposited either in tlakes at the bottom of the cavity, or as false membrane lining its walls, or in the form of interstitial jelly diffused through the meshes of cellular tissue, as follows, eight ounces $=3840$ grains; every 100 grains of which furnish 0.27 of a grain of fibrine, so that the total deposition will amount to $10 \cdot 36$ grains. But we are putting the most extreme case, in supposing the specific gravity of the fluid to be 1029. It will, probably, much oftener be found of specific gravity 1015, or even lower than this. The quantity of albumen then will be not nearly so much as we have assumed, and we shall, therefore, not have nearly so much fibrine to account for. I question whether any one will, unless after careful examination, take upon himself to assert, that in a hydrocele containing eight ounces of fluid, there are not five or six grains of fibrine deposited in one or other of the methods pointed out*.

- I have found by actual experiment, that where the specific gravity of a dropsical effusion does not exceed 1020, it does not contain above 1.6 per cent. of albumen, which would reduce the quantity of fibrine to be accounted for in the above case to about two grains. We must recollect, that we cannot by a simple rule of proportion come at the quantity of albu- 
The above is a hypothetical case, but I can instance one of actual and recent occurrence, which may perhaps be more satisfactory. A young wo. man with enlarged heart, the consequence of rheumatic fever, became the subject of anasarcous swellings of the legs, and of general dropsy of the abdomen. Her difficulties increasing upon her, it was thought advisable that she should be tapped, and the operation was performed on the 9th instant, (Nov. 1830). About twenty pounds of serous fluid were drawn off, of a greenish yellow colour, and transparent. Several flocculent masses of fibrine also passed the canula, and sank to the bottom of the vessel in which the stream was received; this fluid had a specific gravity of 1015, at fifty-nine Faht. A coagulum was formed on the application of heat, but much liquid remained even at a boiling temperature. I took 5000 grains of this serum, and by careful boiling, evaporation, and ablution, obtained all its albumen in a coagulated state. This was carefully dried at a low temperature, and the quantity thus men in a liquid of given specific gravity. If we are to calculate, instead of actually collecting the albumen, we must take into account the specific gravity of dry albumen, the consideration of whether any condensation takes place on adding it to water, the question of the degree in which the specific gravity of the serum is increased by the salts it contains, \&c. So that specific gravity, though a valuable indication of the relative quantity of albumen in serous fluids cannot be depended on as a basis for ascertaining its actual quantity. 
collected amounted to sixty grains, or $1.02 \mathrm{per}$ cent.; the whole amount then of albumen in the twenty pounds of serous fluid, was 1175 grains, for which, the average equivalent of fibrine should be 52.87 grains. I make little doubt, that nearly the whole of this amount might have been collected from the flocculent masses of fibrine, some of which passed the canula, and others of which might be reasonably supposed to have gravitated to the bottom of the abdomen. For the rest, it is not going too far to suppose, some deposition either among the viscera or on the peritoneal lining of the cavity.

The above instance renders it highly probable, that there is, generally, in cases of effusion, a considerable portion of fibrine deposited from the fluid poured forth, and I was strongly disposed to consider the rule general, and that Liquor Sanguinis was in all cases effused, so as to afford us a deposition of fibrine corresponding with the albumen met with in such effusion. Subsequent experiment, however, obliges me to alter this opinion, and to believe that where there is continued effusion, an actual secretion sometimes takes place of albuminous fluid. The following cases are in illustration of this fact.

Eleven days after tapping the young woman whose case has been adduced, she was found to 
be filling again very fast. A few ounces of blood were taken from the arm, and this blood was found to contain 0.319 per cent. which is considerably above the average of fibrine, and only 3.51 per cent., which is equally below the average quantity of albumen; her serum had a specific gravity of 1023. I have no doubt that these undue proportions of fibrine and albumen were owing to a secretion into the cavity of the abdomen of albuminous fluid, without a corresponding deposition of fibrine.

The following instance illustrates the same fact. A woman of forty-eight years of age, who for ten weeks had complained of pains in her loins, anasarcous swelling of her legs, and general debility, was found to be passing urine which was in a high degree coagulable. It was clear, therefore, that albumen was thus abstracted from the blood, and it was a question whether any fibrinous deposition would account for the corresponding constituent of the Liquor Sanguinis. I examined her blood as drawn from the arm, and found it to contain 0.43 per cent. of fibrine, which is much beyond the usual average, and only 1.61 per cent. of albumen. The specific gravity of the serum was only 1020 at 60 . I have, moreover, examined the specific gravity of serum in several cases with which Dr. Bright has furnished me, where patients have passed coagulable urine, and have always found it much below the usual standard; and although my 
attention was not at the time directed to the relative proportions of albumen and fibrine, yet the specific gravity alone sufficiently indicated the deficiency of the former.

It is obviously of importance, in examining the truth of these observations, to note particularly not merely the quantity of an effused fluid, but likewise its specific gravity, which I have elsewhere observed * to mark with sufficient accuracy the relative quantity of albumen it contains. In the instance already adduced of effusion into the ventricles of the brain, we have seen that the quantity of fluid may be considerable, and yet little fibrine will be found in the vicinity. When we come, however, to examine the specific gravity of the effusion, or to test its power of coagulation by the application of heat, we shall find that scarcely any albumen is contained in it.

So in the case of ovarial dropsy, the specific gravity of the fluid contained in the cyst will be low where its walls are thin, and on the contrary loaded with albumen where in those walls we may recognize the corresponding equivalent of fibrine.

In the amnion, where there is little fibrine ne-

- See Paper in the Medical and Chirurgical Transactions, Vol. XVI. Part I. p. 57. 
cessary to form so thin a membrane, there is also little albumen to be found in the fluid which it contains.

I have had one opportunity of examining the fluid from hydatids of the abdomen; its specific gravity was 1004, and it did not contain a trace of albumen even when evaporated to dryness. By the specific gravity in this case, we are furnished with a practical method of distinguishing between dropsy arising from hydatids, and general ascites. We are also thus furnished with another proof, if such were wanting, that these are independent animals, siace the fluid they contain does not resemble the morbid effusions of those cavities in which they are found.

There is one circumstance which will probably strike every one on considering the constitution of the Liquor Sanguinis, as above stated. I mean its great resemblance to the chyle. Like it, the chyle separates into a fluid and a clot, the latter of which is nearly colourless. The nature of that clot is fibrinous in both cases, while the clear fluid is alike albuminous in both. The only apparent difference between Liquor Sanguinis and chyle as described by authors, is, the existence in the latter of an oily material. In a late paper read to the Society, I shewed that in the blood itself, an oil is always to be found, and its presence I conceive vol. XVI. 
to depend on its previous existence in the chyle. The principal difference then between chyle and blood, is, the existence of red particles in the latter, which it is evident are not derived from the former. We must therefore seek their formation in some of the viscera connected with the circulation. The spleen has naturally enough been looked to as their secreting organ, but I have examined with the most accurate and powerful microscopes the blood of a dog, whose spleen had been removed several months previously, and on comparing it with that of a healthy animal, it did not appear deficient in the quantity of its red particles. Whether the liver be destined to this office is a question which eannot be determined in the same manner, and so with respect to the other glandular viscera; but if we can certainly know that the red particles do not enter the circulation with the Liquor Sanguinis, and are not formed by the spleen, we at least narrow the limits of our ignorance, and make some advance towards the ascertainment of their probable source.

The foregoing observations I submit to the indulgent attention of the Society. How far they or the simple experiments on which they are founded may be new, I will not pretend to determine, being only able with certainty to affirm that they are, as far as I know, original with me. The question of their truth, however, is one of much 
greater importance, and I shall be happy if they receive verification from the concurrent testimony of others. Should they, however, only lead by their examination and refutation to a more intimate acquaintance with the subject to which they refer, I shall still consider them as not entirely failing in utility. 